\title{
BMJ Open Weaning from mechanical ventilation in people with neuromuscular disease: protocol for a systematic review
}

\author{
Saint Clair Gomes Bernardes Neto (D) , ${ }^{1,2}$ Rodrigo Torres (D) , ${ }^{3}$ íllia Lima (D) , ${ }^{4}$ \\ Vanessa R Resqueti (D) ,' Guilherme A F Fregonezi (D) ${ }^{1,2}$
}

To cite: Bernardes Neto SCG, Torres R, Lima Í, et al. Weaning from mechanical ventilation in people with neuromuscular disease: protocol for a systematic review. BMJ Open 2019;9:e029890. doi:10.1136/ bmjopen-2019-029890

- Prepublication history and additional material for this paper are available online. To view these files, please visit the journal online (http://dx.doi. org/10.1136/bmjopen-2019029890).

Received 12 March 2019 Revised 09 September 2019

Accepted 17 September 2019

Check for updates

(c) Author(s) (or their employer(s)) 2019. Re-use permitted under CC BY-NC. No commercial re-use. See rights and permissions. Published by BMJ.

${ }^{1}$ RENORBI0 - Biotechnology, Universidade Federal do Rio Grande do Norte, Natal, Brazil ${ }^{2}$ PneumocardioVascular LAB, Hospital Universitário Onofre Lopes, Empresa Brasileira de Serviços Hospitalares (EBSERH) \& Laboratório de Inovação Tecnológica em Reabilitação. Departamento de Fisioterapia, Universidade Federal do Rio Grande do Norte, Natal, RN, Brazil

${ }^{3}$ Physiotherapy, University of Chile, Santiago, Chile

${ }^{4}$ Faculdade de Ciências da Saúde do Trairi - FACISA,

Universidade Federal do Rio Grande do Norte, Santa Cruz, $\mathrm{RN}$, Brazil

Correspondence to

Saint Clair Gomes Bernardes Neto; netosam@gmail.com

\section{ABSTRACT}

Introduction Neuromuscular diseases (NMD) are characterised by progressive muscular impairment. The muscle weakness is directly related to respiratory muscles weakness, causing reduction in vital capacity, especially when associated with mechanical ventilation (MV). Conventional MV weaning in NMD is generally difficult. Weaning process can be conducted in protocols such as: ' $T$ ' piece or Pressure Support Ventilaton. Weaning failure is frequent because of muscle weakness. Protocol aim is to assess the effects of different weaning protocols in NMD patients receiving invasive $\mathrm{MV}$ in weaning success rate, duration of weaning, intensive care unit (ICU) stay, hospital stay and ICU mortality.

Methods and analysis A search will be carried in the Cochrane Neuromuscular Specialised Register, MEDLINE, EMBASE, Web of Science, Scopus, United States National Institutes of Health Clinical Trials Registry, ClinicalTrials. gov and WHO International Clinical Trial Registry Protal, of randomised controlled trials (RCTs) and quasi-RCTs. Inclusion criteria of individuals are adults (above 16 years old) and children (from 5 to 16 years old), with clinical diagnosis of NMD (muscular dystrophy, amyotrophic latera sclerosis, congenital myasthenia, myasthenia gravis, congenital myopathy, spinal muscular atrophy, Guillian Barré Syndrome, severe inherited neuropathies, metabolic myopathies, inflammatory myopathies, mitochondrial diseases) of any gender. All patients ventilated for at least 48 hours due to respiratory failure and clinically considered ready for weaning. Other respiratory or cardiovascular diagnosis associated will not be included. Intervention assessed will be weaning from MV using a protocol with 30 min to 2 hours of spontaneous breathing trial at the end point. All comparisons of different protocols will be considered.

Ethics and dissemination Formal ethical approval is not required as primary data will not be collected, since it will be a systematic review. All studies included should have ethical committee approval. The results will be disseminated through a peer-reviewed publication and in conferences and congresses or symposia.

PROSPERO registration number CRD42019117393.

\section{INTRODUCTION}

Neuromuscular disease (NMD) can be defined as a chronic and progressive disease, which may present with different clinical

\section{Strengths and limitations of this study}

This study will help to identify the best way to conduct mechanical ventilation (MV) weaning in patients with neuromuscular diseases (NMD), improving the outcomes of this population when using MV.

- It will be difficult to find articles that meet the inclusion criteria leading to greater difficulty for statistical analysis.

- There are very different approaches in the weaning process of patients with NMD, and that will bring difficult to compare the protocols.

- Too many NMD will need to be included because of NMD heterogeneity.

characteristics, in which its pattern is based on the location where the injury occurs in a motor unit. ${ }^{12}$ NMD are characterised by progressive muscular impairment, with difficulty in ambulation, swallowing and ventilation, with progressive reduction of vital capacity and increased work of breathing. ${ }^{3}$ These changes lead to the development of chronic respiratory insufficiency, which is an important cause of prolonged ventilatory dependence. $^{4}$

Muscle weakness is directly related to weakness of respiratory muscles, especially the diaphragm. Diaphragmatic weakness, often found in patients with NMD causes a reduction in the capacity to generate force, especially when associated with the use of controlled mechanical ventilation. ${ }^{5}$

Intensive care unit (ICU) admission, regardless of the presence of NMD, may be a cause of neuromuscular disorders that lead to muscle impairment. ${ }^{6}$ It is estimated that such a condition occurs in up to $62 \%$ of critically ill patients in the ICU. ${ }^{7}$ The NMD patients experience this respiratory impairment, in general, by a large proportion of motor units that innervate the respiratory muscles affected. $^{2}$ 
Some risk factors such as use of sedatives, malnutrition, systemic inflammation and prolonged mechanical ventilation may further impair the neuromuscular performance of people admitted to ICU. ${ }^{8}$

The majority of critically ill patients admitted to ICU require ventilatory support for acute or chronic respiratory failure, ${ }^{3}$ specially the NMD ones. In addition, the pattern of neuromuscular abnormalities associated with critical illness, defined as ICU-acquired weakness (ICUAW) ${ }^{4}$ can lead to prolonged mechanical ventilation, a longer hospital stay and increased ventilation. ${ }^{4}$

The emergence of respiratory symptoms, with progressive hypercapnia, can lead to death from respiratory failure. ${ }^{3}$ Long-term invasive or non-invasive mechanical ventilation is the main intervention for people who present with acute respiratory acidosis; progressive decline in vital capacity $(<10-15 \mathrm{~mL} / \mathrm{kg})$; or progressive decline in maximal inspiratory pressure $\left(<20-30 \mathrm{cmH}_{2} \mathrm{O}\right) .{ }^{39}$

Weaning from mechanical ventilation is the process of transition to spontaneous ventilation. ${ }^{10}$ In people with NMD, conventional weaning is generally not possible. ${ }^{11}$

Weaning difficulty may occur in different populations, such as elderly with prolonged ICU hospitalisation, people with chronic respiratory diseases or NMD. ${ }^{12}$ Therefore, the decision to progress to extubation is more challenging in this group of people with advanced respiratory muscle weakness, and this can lead to a need for tracheostomy and prolonged mechanical ventilation. ${ }^{4}$

Difficult weaning can be defined as the requirement of up to three spontaneous breathing trials (SBT) in a period of no longer than 7 days of mechanical ventilation to achieve extubation. ${ }^{1013}$

The weaning process may be conducted in different protocols such as the following:

- 'T' piece: in which the patient receives only supplemental oxygen through a T-shaped tube connected to an endotracheal tube (orotracheal or tracheostomy). ${ }^{10}$

- Continuous positive airway pressure (CPAP): the weaning protocol involves using a continuous pressure, equal to the previous positive end-expiratory pressure level used before. ${ }^{10}$

- Pressure support: the use of progressive lower levels of inspiratory pressure support until it reaches 5-8 $\mathrm{cmH}_{2} \mathrm{O} .^{10}$

Successful weaning is defined as the ability to maintain spontaneous ventilation without the need for reintubation and invasive mechanical ventilation for 48 hours after extubation. ${ }^{10}$ For patients with NMD, due to the difficulty of weaning, it may be also defined as the absence of a need for tracheostomy and mechanical ventilation for 5 days after extubation. $^{4}$

Postweaning monitoring should observe whether two of the following findings are present: respiratory acidosis ( $\mathrm{pH}<7.35 ; \mathrm{PaCO}_{2}>45 \mathrm{~mm} \mathrm{Hg}$ ); $\mathrm{SpO}_{2}<90 \%$ or $\mathrm{PaO}_{2}<60$ $\mathrm{mm} \mathrm{Hg}$ with $\mathrm{FiO}_{2}>50 \%$; i $>35 \mathrm{rpm}$; decreased level of consciousness, restlessness or excessive sweating; or signs suggestive of respiratory muscle fatigue, such as the use of accessory muscles or paradoxical movement of the abdomen, in order to determinate the need to re-establish mechanical ventilation again. ${ }^{410}$

Weaning failure from invasive ventilation is frequent in people with NMD due to muscle weakness and gradual hypercapnia. ${ }^{4}$ In this way, the non-invasive ventilation, even after weaning failure, is an option. And a future weaning can be conducted when and if clinically possible. ${ }^{412}$ Although this whole process significantly increases health costs with this patient population.

\section{Objectives}

The aim of this systematic review is to assess the effects of different weaning protocols in people with NMD receiving invasive mechanical ventilation. Our secondary aim is to assess how the different protocols affect weaning success, duration of weaning, duration of stay in the ICU, duration of hospital stay, ICU mortality and also to assess adverse effects.

\section{METHODS}

\section{Eligibility criteria}

Studies will be selected according to the criteria outlined below.

\section{Study designs}

We will include randomised controlled trials (RCTs) and quasi-RCTs (experimental study with participants subjected to some type of intervention or control group, and with the same outcome of interest measured. But in this kind of study, also known as non-randomised trial, populations are subjected to any of the groups using other methods of allocating, usually not truly random). Other study types, such as non-randomised trials, crossover studies and casecontrol studies will be described in the 'Discussion' section of the review, but they will not be included in the Results section. We will include studies reported as full-text, those published as abstract only and unpublished data. There will be no restrictions as to language.

\section{Participants}

We will consider for inclusion adults (above 16 years old) and children (from 5 to 16 years old) people with a clinical diagnosis of a NMD (muscular dystrophy of any origin including Duchenne muscular dystrophy, amyotrophic lateral sclerosis, congenital myasthenia, myasthenia gravis, congenital myopathy, spinal muscular atrophy, Guillian Barré Syndrome, severe inherited neuropathies, metabolic myopathies (Pompe disease), inflammatory myopathies and mitochondrial diseases) of any gender.

We will consider all patients ventilated for at least 48 hours with orotracheal tube or tracheostomy because of acute respiratory failure, and considered by physicians to be ready for weaning according to clinical 
criteria and weaning parameters. No patients with other respiratory or cardiovascular clinical diagnosis associated will be included, nor patients with mixed NMD diagnosis.

If any subset of participants with NMD is analysed, these patients will be included.

\section{Interventions}

The intervention assessed will be the process of weaning from mechanical ventilation in people with NMD using a protocol with criteria for deciding if the patient is ready for extubation with $30 \mathrm{~min}$ to 2 hours SBT at the end point of the protocol.

We will consider the following protocols for inclusion.

1. Pressure support ventilation, with gradual reduction of the support pressure.

2. Synchronised intermittent mandatory ventilation, with gradual reduction of respiratory rate and support pressure.

3. CPAP, with gradual reduction of applied pressure.

4. ' $T$ ' piece, with progressive increase of spontaneous ventilation time.

\section{Comparators}

We will consider any comparisons of the different protocols.

The protocols will also be compared in relation to the classification of weaning outcomes, in order to identify which protocols develop better outcomes.

- Simple-successful after first attempt.

- Difficult-require up to three attempts (or less than 7 days to reach success).

- Prolonged-require more than 7 days to reach success.

\section{Outcomes}

\section{Primary outcome}

Weaning success is defined as the ability to maintain spontaneous ventilation without the need for reintubation and invasive mechanical ventilation for 48 hours after extubation. $^{10}$

\section{Secondary outcomes}

- Duration of weaning in patients with acute and prolonged mechanical ventilation-defined as the time between the weaning protocol initiation and the moment of extubation.

- Duration of ICU stay in patients with acute and prolonged mechanical ventilation-defined as the time between ICU admission and ICU discharge.

- Duration of hospital stay in patients with acute and prolonged mechanical ventilation-defined as the time between hospital admission and hospital discharge.

- ICU mortality rate in patients with acute and prolonged mechanical ventilation-defined as the mortality rate during ICU stay.

- Incidence of pneumothorax during mechanical ventilation period.
Incidence of ventilation associated pneumonia.

\section{Language}

We will include articles reported in English and other languages. There will be no restrictions.

\section{Information sources}

\section{Electronic searches}

We will search the Cochrane Neuromuscular Specialised Register (The Cochrane Library, current issues), MEDLINE, EMBASE, Web of Science and Scopus. We will scan conference abstracts for relevant studies.

We will also search the United States National Institutes of Health Clinical Trials Registry, ClinicalTrials.gov (ClinicalTrials.gov) and the WHO International Clinical Trials Registry Portal (apps.who.int/trialsearch/).

We will search all databases from January 2009 to December 2019, and we will impose no restriction on language of publication.

We will identify non-randomised studies for inclusion in the discussion from the same search results.

We will search reference lists of all relevant and included trials and review articles for additional references. We will search for errata or retractions of included trials. We will also search relevant manufacturers' websites for trial information. And we will search grey literature, in reports of technical research and projects related to government programme, to identify other studies.

We will contact study authors of included trials to identify additional trials whether published or unpublished.

If no RCTs or quasi-RCTs in this area are not found, the authors will review other well-designed observational studies, where the population (NMD), intervention (mechanical ventilation weaning) and outcome (weaning success) are clearly documented, in the 'Discussion' section of the review. We will identify these (nonrandomised studies) via a search in MEDLINE (from inception to the present), EMBASE (from inception to the present), Web of Science (from inception to the present) and Scopus (from inception to the present). This will be done in order to give a comprehensive descriptive narrative of any non-randomised data.

\section{Search strategy}

Search terms will include: 'neuromuscular disease' or all other terms compatible with clinical diagnoses of these types of diseases, such as 'muscular dystrophy', 'Duchenne muscular dystrophy', 'amyotrophic lateral sclerosis', 'congenital myasthenia', 'myasthenia gravis', 'congenital myopathy', 'spinal muscular atrophy', 'Guillian Barré Syndrome', 'severe inherited neuropathies', 'metabolic myopathies', 'Pompe disease', 'inflammatory myopathies' and 'mitochondrial diseases' combined with 'mechanical ventilation' or 'artificial respiration' or 'mechanical ventilation weaning' or 'ventilator weaning' or 'respirator weaning' and all the combination between them.

An example of the search strategy is available as a online supplementary file. 


\section{Study records}

\section{Selection of studies}

Two review authors (SCBN and RTC) will independently screen titles and abstracts of all the potential studies retrieved by the search for inclusion and code them as 'retrieve' (eligible or potentially eligible/unclear) or 'do not retrieve'. We will identify and exclude duplicates and collate multiple reports of the same study so that each study rather than each report is the unit of interest in the review. We will retrieve full-text study reports/publications, and two review authors (SCBN and RTC) will independently screen the full text and identify studies for inclusion, and identify and record reasons for exclusion of the ineligible studies.

We will resolve any disagreements through discussion or, if required, through consultation with a third review author (GAFF).

We will report the selection process in sufficient detail to complete a Preferred Reporting Items for Systematic Review and Meta-Analysis Protocols flow diagram and 'Characteristics of excluded studies' table.

\section{Data extraction and management}

We will use a data extraction form that we will initially pilot on at least one trial included in the review to collect study characteristics and outcome data. One review author (SCBN) will extract study characteristics from included trials. We will collect information on study design and setting, participant characteristics (including disease severity and age), study eligibility criteria, details of the intervention(s) given, the outcomes assessed, the source of study funding and any conflicts of interest stated by the investigators.

Two review authors (SCBN and RTC) will independently extract outcome data from included trials. We will note in the 'Characteristics of included studies' table if the trials did not report outcome data in a usable way. We will resolve any disagreements by consensus or consult a third review author (GAFF). One review author (SCBN) will transfer data into Review Manager (RevMan) V.5.3. ${ }^{14}$ A second review author (RTC) will check the outcome data entries.

The same review author (RTC) will spot-check study characteristics for accuracy against the trial report. When reports require translation, the translator will extract data directly using a data extraction form. To minimise bias in the review process, the review authors will not screen studies for inclusion, extract data, or assess the risk of bias in trials they themselves have authored. In such circumstances, we will involve a third review author (GAFF).

\section{Risk of bias individual studies}

Two review authors (SCBN and RTC) will independently assess risk of bias for each study using the criteria outlined in the Cochrane Handbook for Systematic Reviews of Interventions. ${ }^{15}$ These authors will resolve disagreements by discussion or by involving another review author (GAFF).
We will assess the risk of bias according to the following domains:

1. Random sequence generation.

2. Allocation concealment.

3. Blinding of participants and personnel.

4. Blinding of outcome assessment.

5. Incomplete outcome data.

6. Selective outcome reporting.

7. Other bias.

We will grade each potential source of bias as high, low or unclear and provide a quote from the study report together with a justification for our judgement in the 'Risk of bias' table. We will summarise the risk of bias judgements across different studies for each of the domains listed. We will consider blinding separately for different key outcomes where necessary (eg, for unblinded outcome assessment, risk of bias for all-cause mortality may be very different than for a patient-reported pain scale). Where information on risk of bias relates to unpublished data or correspondence with a trialist, we will note this in the 'Risk of bias' table. When considering treatment effects, we will take into account the risk of bias for the studies that contribute to that outcome.

If we are able to pool a sufficient number of studies, that is, more than 10 trials, ${ }^{15}$ we will create and examine a funnel plot to explore possible small study biases.

\section{Data synthesis}

\section{Measures of treatment effect}

We will analyse dichotomous data as risk ratios (RR) with corresponding 95\% CI and continuous data as mean difference (MD) with $95 \%$ CI, or as standardised mean difference with $95 \%$ CI for results across studies with outcomes that are conceptually the same but measured in different ways. We will enter data presented as a scale with a consistent direction of effect.

We will undertake meta-analyses only where this is meaningful, that is if the treatments, participants and the underlying clinical question are similar enough for pooling to make sense. This will be identified if there are two or more trials with comparable populations and interventions.

Where a single trial reports multiple trial arms, we will include only the arms relevant to the review question.

All data will be pooled according to age group, dividing them into two groups (adults-over 16 years old, and children-between 5 and 16 years old). After this grouping, the analysis will be done, first, comparing the success rate and failure rate in each of the groups. Subsequently, the data will also be evaluated taking into consideration the weaning outcomes in simple, difficult and prolonged (as described in the types of interventions).

\section{Unit of analysis issues}

We do not expect to have any crossover or cluster randomised controlled trials, since weaning is a one-off event and also due to the lack of control group, since all 
patients are submitted to the same intervention, which is weaning from mechanical ventilation.

If we are able to find cluster randomised controlled trials with different clusters of different NMD, we will conduct this analysis.

\section{Assessment of heterogeneity}

We will use the $\mathrm{I}^{2}$ statistic to measure heterogeneity among the trials in each analysis. If we identify substantial unexplained heterogeneity, we will report randomeffects results and explore possible causes by prespecified subgroup analysis.

We will be following the rough guide to interpretation outlined in the Cochrane Handbook for Systematic Reviews of Interventions.

- 0\%-40\%: might not be important;

- 30\%-60\%: may represent moderate heterogeneity;

- 50\%-90\%: may represent substantial heterogeneity and

- 75\%-100\%: considerable heterogeneity.

\section{Data synthesis}

If the review includes more than one comparison that cannot be included in the same analysis, we will report the results for each comparison separately.

\section{'Summary of findings' table}

- We will create a 'Summary of findings' table using the following outcomes.

- Weaning success.

- Duration of weaning (time difference between weaning protocol initiation and the moment of extubation moment).

- Duration of ICU stay.

- Duration of hospital stay.

- ICU mortality rate in patients with acute and prolonged mechanical ventilation-defined as the mortality rate during ICU stay.

- Incidence of pneumothorax during mechanical ventilation period.

- Incidence of ventilation associated pneumonia.

We will use the five Grading of Recommendatons Assessment, Development and Evaluaton (GRADE) considerations (study limitations, consistency of effect, imprecision, indirectness and publication bias) to assess the quality of a body of evidence (studies that contribute data for the prespecified outcomes). We will use methods and recommendations described in the Cochrane Handbook for Systematic Reviews of Interventions ${ }^{15}$ using GRADEpro software (GRADEpro GDT). We will justify all decisions to downgrade or upgrade the quality of studies using footnotes, and we will make comments to aid readers' understanding of the review where necessary. Two authors will independently grade the quality of the evidence. They will resolve disagreements by discussion and by consultation with a third review author.

\section{Subgroup analysis and investigation of heterogeneity}

- We plan to perform the following subgroup analyses.
- Simple weaning: successful after first attempt.

- Difficult weaning: require up to three attempts.

- Prolonged weaning: require more than 7 days to reach success.

- Children: from 5 to 16 years old.

- Adults: above 16 years old.

We will use both primary and secondary outcome measures in all subgroup analyses. We will use the formal test for subgroup interactions in Review Manager V.5.3. ${ }^{14}$

\section{Sensitivity analysis}

We plan to undertake the following sensitivity analyses.

3 wRepeat the analysis by excluding studies at high risk of bias (sequence generation, allocation concealment, blinding of personnel, outcome assessment and attrition).

If there are one or more very large trials, we will repeat the analysis by excluding them to examine how much they dominate the results.

\section{Reaching conclusions}

We will base our review conclusions only on findings from the quantitative or narrative synthesis of included trials. We will avoid making recommendations for practice. Our implications for research will suggest priorities for future research and outline the remaining uncertainties in the area.

\section{Patient and public involvement}

In the present protocol of systematic review and in the subsequent systematic review, there will be no involvement of patients or public.

The paper proposes to use results previously authorised and published by other authors, without there being any need for patient or public involvement. The research question was developed based on the questions raised by other authors, most of the time according to the clinical difficult and necessity of improving the weaning protocols for this population.

The results of the present study will be published in indexed journal so it can be available for NMD patients, in general, and public, specially health professionals.

\section{CONCLUSION}

This systematic review will provide evidence in different weaning protocols that can be applied to the NMD patients, analysing the weaning success rate, leading to extubation. The hypothesis is that one specific protocol has higher success weaning rates.

Where sufficient data are available, we will conduct a meta-analysis to confirm the relationship between the different protocols and duration of weaning, duration of stay in the ICU, duration of hospital stay and ICU mortality. It will also be able to assess adverse effects of weaning protocols that fail to lead to extubation.

Moreover, if the hypothesis is confirmed, the review will clarify the reasons any weaning strategy interfere to higher success weaning rates. 
Overall, the review will complement the evidence based on mechanical ventilation weaning for NMD patients.

Contributors SCBN: screen titles, abstracts and full text to identify studies for inclusion or exclusion; extract study characteristics; extract outcome data; transfer data into RevMan; assess risk of bias. RTC: screen titles, abstracts and full text to identify studies for inclusion or exclusion; extract outcome data; check outcome data entries; spot-check study characteristics for accuracy; assess risk of bias. IL: development of the text; statistical analysis and revision of the final text. VRR: development of the text; statistical analysis, revision of the final text. GAFF: discussion about the disagreements the two authors have in any issues; screen studies the other two authors have authored.

Funding Guilherme Augusto de Freitas Fregonezi received a grant from CNPq number 312876/2018-1, and Vanessa Resqueti received a grant from CNPq number 315580/2018-6.

Competing interests None declared.

Patient consent for publication Not required.

Provenance and peer review Not commissioned; externally peer reviewed.

Open access This is an open access article distributed in accordance with the Creative Commons Attribution Non Commercial (CC BY-NC 4.0) license, which permits others to distribute, remix, adapt, build upon this work non-commercially, and license their derivative works on different terms, provided the original work is properly cited, appropriate credit is given, any changes made indicated, and the use is non-commercial. See: http://creativecommons.org/licenses/by-nc/4.0/.

\section{ORCID iDs}

Saint Clair Gomes Bernardes Neto http://orcid.org/0000-0001-5089-0564

Rodrigo Torres http://orcid.org/0000-0001-7974-4333

illia Lima http://orcid.org/0000-0002-0301-4702

Vanessa R Resqueti http://orcid.org/0000-0003-4817-9364

Guilherme A F Fregonezi http://orcid.org/0000-0003-4938-7018

\section{REFERENCES}

1 Anziska Y, Sternberg A. Exercise in neuromuscular disease. Muscle Nerve 2013;48:3-20.
2 Rezania K, Goldenberg FD, White S. Neuromuscular disorders and acute respiratory failure: diagnosis and management. Neurol Clin 2012;30:161-85.

3 Ambrosino N, Carpenè N, Gherardi M. Chronic respiratory care for neuromuscular diseases in adults. Eur Respir J 2009;34:444-51.

$4 \mathrm{Kim}$ SM, Kang S-W, Choi Y-C, et al. Successful extubation after weaning failure by noninvasive ventilation in patients with neuromuscular disease: case series. Ann Rehabil Med 2017;41:450-5

5 Vassilakopoulos T, Petrof BJ. Ventilator-Induced diaphragmatic dysfunction. Am J Respir Crit Care Med 2004;169:336-41.

6 Bach JR, Gonçalves MR, Hamdani I, et al. Extubation of patients with neuromuscular weakness. Chest 2010;137:1033-9.

7 Epstein SK, Ciubotaru RL. Independent effects of etiology of failure and time to reintubation on outcome for patients failing extubation. Am J Respir Crit Care Med 1998;158:489-93.

8 Hermans G, Van den Berghe G. Clinical review: intensive care unit acquired weakness. Critical Care 2015;19:274-83.

9 Chevrolet JC, Deléamont P. Repeated vital capacity measurements as predictive parameters for mechanical ventilation need and weaning success in the Guillain-Barré syndrome. Am Rev Respir Dis 1991;144:814-8.

10 Zein H, Baratloo A, Negida A, et al. Ventilator weaning and spontaneous breathing trials; an educational review. Emerg 2016;4:65-71.

11 Fan E, Cheek F, Chlan L, et al. An official American thoracic Society clinical practice guideline: the diagnosis of intensive care unit-acquired weakness in adults. Am J Respir Crit Care Med 2014;190:1437-46.

12 Tsara V, Moisiadis N, Antoniadou M, et al. Characteristics and outcome of patients with difficult weaning from mechanical ventilation: an 18 years' experience of a respiratory intermediate unit attached to a pulmonary department. Hippokratia 2015;19:37-40.

13 Sklar MC, Burns K, Rittayamai N, et al. Effort to breathe with various spontaneous breathing trial techniques. A physiologic meta-analysis. Am J Respir Crit Care Med 2017;195:1477-85.

14 The Cochrane Collaboration. Review Manager (RevMan) [Computer program]. Version 5.3. Copenhagen The Nordic Cochrane Centre; 2014.

15 Higgins JPT, Green S, The Cochrane Collaboration. Cochrane Handbook for systematic reviews of interventions. version 5.1.0, 2011. Available: www.cochrane-handbook.org 